\title{
Distribución del tamaño de partícula por dispersión dinámica de luz de la tetracarboxifenilporfirina de cobre (II) anclada al dióxido de titanio
}

\section{Particle size distributions by dynamic light scattering of copper (II) tetracarboxyphenilporphyrn anchored on titanium dioxide}

\author{
Carlos Enrique Díaz Uribe \\ Ph. D. Química. Profesor Asistente, \\ Universidad del Atlántico, Colombia \\ carlosdiaz@mail.uniatlantico.edu.co
}

\author{
William A. Vallejo \\ Ph. D. Ciencias Químicas, Profesor Asociado, \\ Universidad del Atlántico, Colombia \\ williamvallejo@mail.uniatlantico.edu.co
}

\author{
Esneyder Puello Polo \\ Ph. D. Ciencias: Química, Profesor Asistente, \\ Universidad del Atlántico, Colombia \\ esneyderpuello@mail.uniatlantico.edu.co
}

\begin{abstract}
Resumen- La sensibilización del dióxido de titanio con porfirinas es esencial para la generación de especies reactivas del oxígeno inducida con luz visible. Sin embargo, estos sensibilizadores tienden a lixiviarse en el medio de reacción. La distribución del tamaño de partícula es una propiedad fundamental para caracterizar sistemas de partículas en suspensión. En este trabajo se estudió la distribución del tamaño de partícula de la tetracarboxifenilporfirina de cobre (II) anclada sobre dióxido de titanio por la técnica de dispersión dinámica de luz. Los resultados indican que la técnica de dispersión dinámica de luz es adecuada para determinar la estabilidad de estos sistemas, mostrando un solo tipo de partícula para el sistema TCPPCu/ $/ \mathrm{TiO}_{2}$ con diámetro promedio de $211,1 \mathrm{~nm}$.
\end{abstract}

Palabras clave- $\mathrm{TiO}_{2}$, Sensibilización, DLS, Tamaño de partícula.

Abstract-The sensitization of titanium dioxide with porphyrins is essential to the generation of reactive oxygen species under irradiation with visible light. However, these sensitizers tend to leach into the reaction medium. The particle size distribution is a critical property for characterizing particles in suspension systems. In this work we determinated the particle size distribution of copper (II) tetracarboxyphenylporphyrin anchored on titanium dioxide by the technique of dynamic light scattering (DLS). The results showed only one type of particle to the system TCPPCu/ $\mathrm{TiO}_{2}$ with average diameter of $211.1 \mathrm{~nm}$ and that DLS is suitable for determining the stability of these systems.

Keywords $-\mathrm{TiO}_{2}$, sensitization, DLS, size of particle.

\section{INTRODUCCIÓN}

Las porfirinas son utilizadas como sensibilizadores debido a su alta absorción en la región visible del espectro electromagnético, estos compuestos son eficientes en las reacciones de oxidación catalítica y generan productos menos tóxicos [1]. Su inmovilización bloquea la formación de agregados, facilita la reutilización del sensibilizador, la separación de los productos de la reacción, y la utilización en diferentes solventes [2], [3]. Una de las matrices más utilizadas para inmovilizar este tipo de compuestos es el dióxido de titanio [4]-[7].

La estabilidad del sensibilizador depende de la fortaleza de la unión al soporte, la preparación, la aplicación general y la estabilidad. Hay un considerable interés en insertar complejos macrocíclicos dentro de materiales amorfos porosos por simple adsorción para diversas aplicaciones [8], [9]. La mayor ventaja de encapsular el sensibilizador es el control del medio de reacción y sus condiciones de preparación [10]. El valor del pH en solución es un parámetro fundamental que previene la lixiviación de un sensibilizador, por esto en este trabajo se estudió la distribución del tamaño de partícula de la tetracarboxifenilporfirina de cobre (II) adsorbida sobre dióxido de titanio a un pH 5 
por la técnica de dispersión dinámica de luz. La técnica de dispersión de luz dinámica permite determinar el tamaño de partículas en suspensión hasta rangos del orden de los nanómetros, el uso del movimiento Browniano de las partículas en suspensión permite alcanzar estos límites de sensibilidad, adicionalmente este método no altera de ninguna manera la matriz de estudio debido a que es una técnica no destructiva. Los resultados indican que la técnica de dispersión dinámica de luz es adecuada para determinar la estabilidad de los sensibilizadores anclados al dióxido de titanio.

\section{METODOLOGÍA}

\subsection{Síntesis y caracterización}

La tetracarboxifenilporfirina sin metal se sintetizó al añadir $30 \mathrm{mmol}$ de pirrol previamente destilado a una mezcla de 4-carboxibenzaldehido (30 $\mathrm{mmol}$ ), ácido propiónico (105 mL) y nitrobenceno (45 mL). La mezcla se calentó a $120^{\circ} \mathrm{C}$ durante 1h. El solvente se removió por destilación al vacío y el sólido obtenido se disolvió con una solución de $\mathrm{NaOH}(0,1 \mathrm{M})$. La porfirina se precipitó con una solución de $\mathrm{HCl}$ 0,1 M, se disolvió en etanol y se cristalizó por evaporación del solvente [11].

La metaloporfirina se preparó colocando a reflujo la TCCPP $(0.33 \mathrm{mmol})$ con cloruro de cobre (II) en cantidades correspondientes a $1,82 \mathrm{mmol}$, en dimetilformamida $(70 \mathrm{~mL})$ durante $2 \mathrm{~h}$. La dimetilformamida se removió por destilación y la TCPPCu se precipitó al adicionar agua. El precipitado se disolvió en una solución de $\mathrm{NaOH}(0.1 \mathrm{M})$ y se recristalizó con una solución de $\mathrm{HCl}(0,1 \mathrm{M})$. Finalmente, la porfirina se filtró y secó a temperatura ambiente.

La TCPPCu/ $/ \mathrm{TiO}_{2}$ se preparó según el siguiente procedimiento: se agregó 0.08438 g de TCPPCu a una mezcla de $0.1 \mathrm{~g}$ de $\mathrm{TiO}_{2}$ Degussa $\mathrm{P}-25 \mathrm{a} \mathrm{pH}=3$ (ajustado con $\mathrm{HNO}_{3}$ ). Se dejó en reflujo por 24 horas, el solvente se rotoevaporó y el sólido se secó a temperatura ambiente [12].

Los espectros UV-vis de la TCPP y TCPPCu en etanol se registraron mediante un espectrofotómetro Hewlett-Packard 8453. Los espectros de reflectancia difusa UV-vis de la porfirina adsorbida se registraron con un espectrofotómetro Lambda 4 Perkin-Elmer equipado con una esfera de inte- gración, mediante sulfato de bario como matriz de referencia.

\subsection{Caracterización del tamaño de partícula por dispersión dinámica de luz}

El tamaño de partícula en suspensión fue determinado mediante la técnica de Dispersión Dinámica de Luz (Espectroscopia de Correlación Fotónica). El equipo utilizado fue un Zetasizer versión 6.2 de Malvern Instruments Ltda. Para el análisis se tomó $1 \mathrm{~g} / \mathrm{L}$ de TCPPCu/ $/ \mathrm{TiO}_{2}$ a pH=5 en agua como dispersante. Todos los experimentos se midieron mediante dispersión dinámica, el cual mide las fluctuaciones de la intensidad de la dispersión en función del tiempo. La distribución de intensidad vista en el programa DLS se obtuvo por el análisis de la función de correlación por el análisis del menor de los cuadrados no negativo.

\section{RESULTADOS Y DISCUSIÓN}

\subsection{Espectrofotometría}

El espectro UV-vis de la TCPP sintetizada se muestra en la Fig. 1., en él se observa la banda de absorción máxima denominada banda Soret a $419 \mathrm{~nm}$ (transición a1u $(\pi)$-eg* $(\pi)$ ), y 4 bandas $Q$ $(514,548,588$ y $645 \mathrm{~nm})$ características de la porfirina no metálica correspondientes a transiciones a2u $(\pi)-\operatorname{eg} *(\pi)$ [13]; estas señales se maximizan dentro de la Fig. 1.

Fig.1. ESPECTRO UV-VIS DE LA TCPP Y DE LA TCPPCU

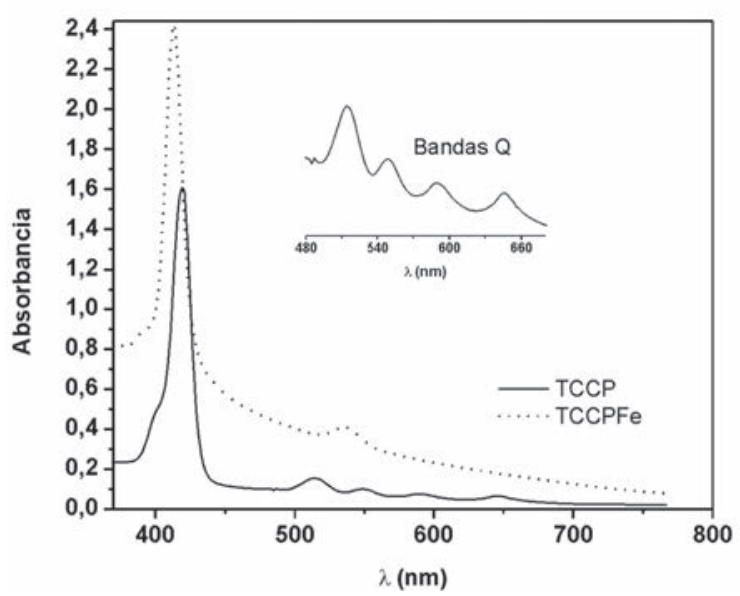

El espectro UV-vis de la TCPPCu (Fig. 1) exhibe la banda Soret a $417 \mathrm{~nm}$ y 1 banda Q en $534 \mathrm{~nm}$. La disminución del número de bandas $\mathrm{Q}$ es típico 
de las metaloporfirinas. Cuando el ión metálico se coordina con los átomos de nitrógeno del macrociclo, la simetría de la molécula se incrementa y el número de bandas $\mathrm{Q}$ decrece [14]. La metalación de las porfirinas produce desplazamiento de la bandas Soret y Q por la interacción del metal con la nube electrónica $\pi$ de los macrociclos. En el espectro de la TCPPCu se observa un desplazamiento de las bandas de absorción hacia el azul, posiblemente debido a la desestabilización de la nube electrónica $\pi$ del ligando inducida por el metal e incremento de la energía de transición [14].

\subsection{Reflectancia difusa}

El espectro de reflectancia difusa de la TCPP$\mathrm{Cu} / \mathrm{TiO}_{2}$ se muestra en la Fig. 2. Las bandas de absorción de TCPPCu adsorbida sobre $\mathrm{TiO}_{2}$ son anchas y de menor intensidad con respecto al espectro de TCPPCU (II), y muestran un corrimiento hacia la región del rojo que puede atribuirse a la distorsión de los anillos de porfirina; el desplazamiento hacia el rojo generalmente se observa cuando las porfirinas son inmovilizadas sobre soportes inorgánicos $[15,16]$. En el caso de la TCPP$\mathrm{Cu}$ adsorbida al $\mathrm{TiO}_{2}$, la banda Soret es desplazada de 417 a $425 \mathrm{~nm}$ y la banda $\mathrm{Q}$ de igual manera es desplazada de 539 a $547 \mathrm{~nm}$, se corrobora que el proceso de inmovilización se ha realizado.

Fig. 2. ESPECTRO DE REFLECTANCIA DIFUSA TCPPCU(II)/TIO

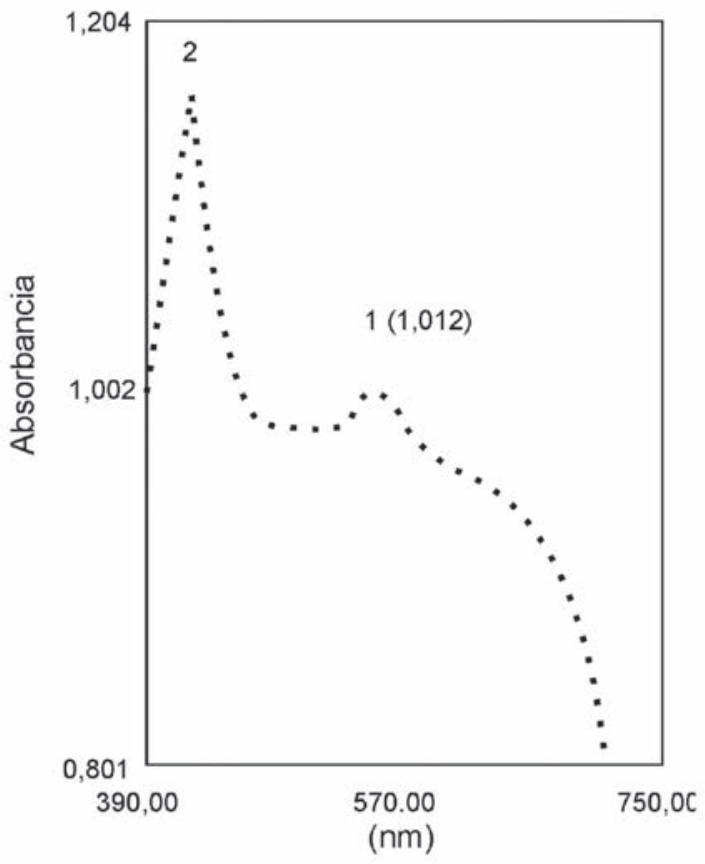

\subsection{Caracterización del tamaño de partícula por dispersión dinámica de luz-DLS}

Para analizar el tamaño de partícula en la solución se tomó $1 \mathrm{~g} / \mathrm{L}$ de $\mathrm{TCPPCu} / \mathrm{TiO}_{2}$ a $\mathrm{pH}=5$ en agua como dispersante. El análisis se realizó mediante dispersión dinámica, el cual mide las fluctuaciones de la intensidad de la dispersión en función del tiempo. La Fig. 3. muestra el coeficiente de correlación de la intensidad de luz dispersada por las partículas de TCPPCu/ $\mathrm{TiO}_{2}$ a pH 5. El punto en que la correlación de la señal empieza a caer proporciona información del diámetro promedio y el ángulo de caída permite observar la 'dispersidad $^{`}$ de la distribución de las partículas. Se obtiene un ángulo similar y una línea de base corta, regular y con poco ruido, lo que permite inferir la baja polidispersidad.

Fig. 3. COEFICIENTE DE CORRELACIÓN DE LA INTENSIDAD DE LUZ DISPERSADA

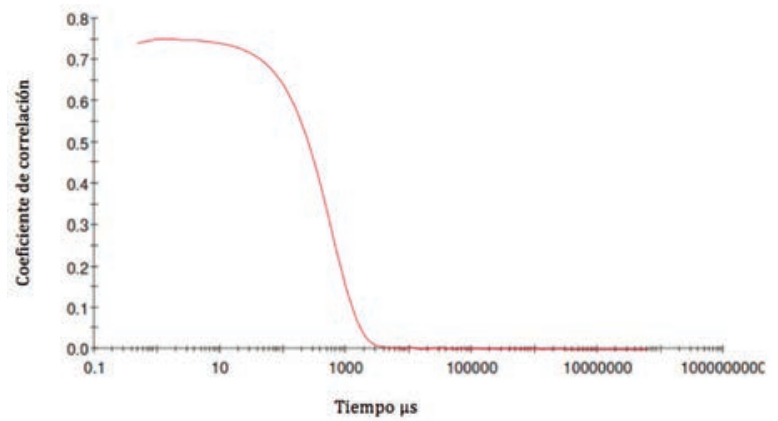

La Fig. 4. muestra la calidad del ajuste de cumulantes (autocorrelación) a los datos medidos indicando que el diámetro calculado y la dispersidad obtenida para las partículas medidas son fiables. En ellas se puede ver que la función de autocorrelación de la intensidad de luz dispersada disminuye más rápidamente después de los $1000 \mathrm{~ms}$.

Fig. 4. FUNCIÓN DE AUTOCORRELACIÓN DE LUZ DISPERSADA

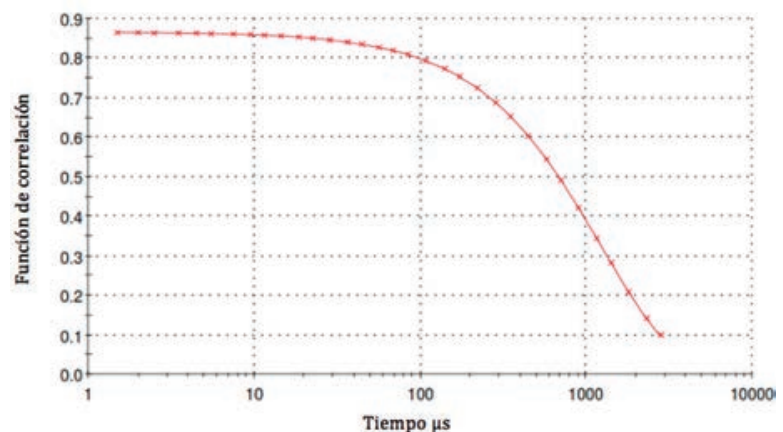


El análisis de DLS correspondiente a la intensidad muestra una banda (Fig. 5), el cual sugiere la formación de un solo grupo de partículas con un diámetro promedio de $211.1 \mathrm{~nm}$.

Fig. 5. ESTUDIO DE DLS EN INTENSIDAD

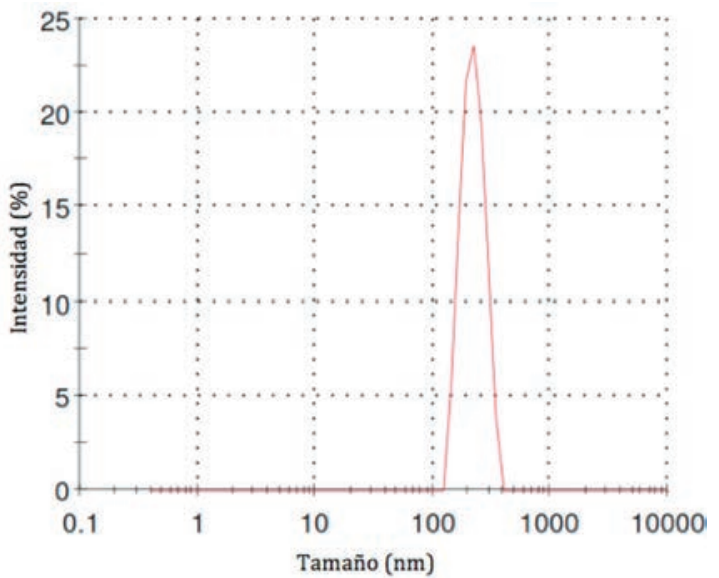

En la Figura 6 se muestra la gráfica del tamaño de partícula en función del número de partículas. Se puede observar un solo tipo de partícula indicando que a este valor de $\mathrm{pH}$ no hay lixiviación de la tetracarboxifenilporfirina.

Fig. 6. DISTRIBUCIÓN DEL NÚMERO DE PARTÍCULAS

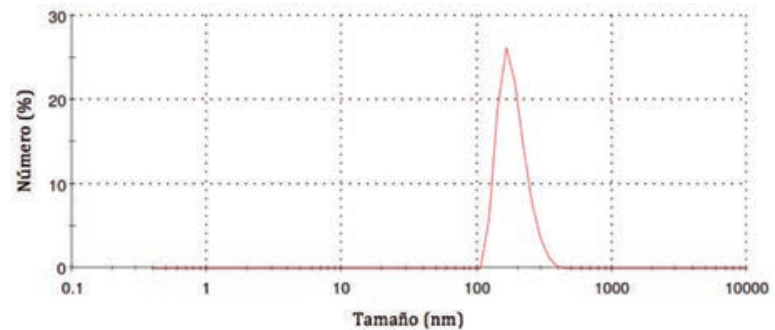

La gráficas de volumen en función del tamaño de partícula confirman que a $\mathrm{pH}=5$ solo hay un mismo tipo de partícula. La Fig. 7. muestra la variación del porcentaje en volumen con respecto al tamaño de las partículas presentes.

Fig. 7. DISTRIBUCIÓN DEL VOLUMEN EN FUNCIÓN DEL TAMAÑO DE PARTÍCULA

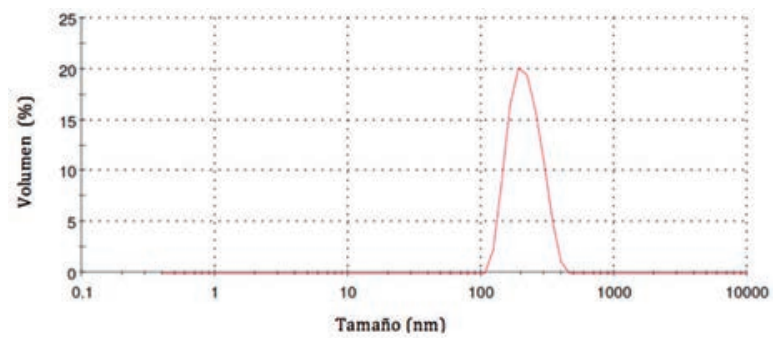

\section{CONCLUSIONES}

Mediante la técnica de dispersión dinámica de luz, se encontró un solo tipo de partícula a $\mathrm{pH}=5$ del sistema tetracarboxifenilporfirina de cobre (II) anclada al dióxido de titanio en dispersión acuosa, esto indica la estabilidad de la porfirina sobre el $\mathrm{TiO}_{2}$ a este valor de $\mathrm{pH}$. Este resultado indica que el sistema TCPPCu/ $\mathrm{TiO}_{2}$ puede ser utilizado en diferentes procesos de fotocatálisis para oxidación de colorantes sin que se presente lixiviación del sensibilizador. Finalmente, los resultados indican que la técnica de dispersión dinámica de luz es adecuada para determinar la estabilidad de estos sistemas.

\section{AGRADECIMIENTOS}

Los autores agradecen al profesor Fernando Martínez Ortega de la Universidad Industrial de Santander por el análisis de DLS.

\section{REFERENCIAS}

[1] M. Hajimohammadi, N. Safari, H. Mofakham, A. Shaabani A, "A new and efficient aerobic oxidation of aldehydes to carboxylic acids with singlet oxygen in the presence of porphyrin sensitizers and visible light," Tetrahedron Lett., Vol. 51, pp. 4061-4065, Agos. 2010.

[2] K.C. Christoforidis, M. Louloudi, E. Milaeva, Y. Deligiannakis, "Mechanism of catalytic decomposition of pentachlorophenol by a highly recyclable heterogeneous $\mathrm{SiO}_{2}$-[Fe-porphyrin] catalyst," J. Catal., vol. 270, pp. 153-162, Mar. 2010.

[3] J.H. Cai, J.W. Huang, H.C. Yu, L.N. Ji, "Fabrication and characterizations of silica composite microspheres immobilized with porphyrins and their photocatalytic properties," Journal of the Taiwan Inst. Chem. E., Vol. 43, pp. 958-964, Agos. 2012.

[4] A. Kathiravan, R. Renganathan, "Effect of anchoring group on the photosensitization of colloidal $\mathrm{TiO}_{2}$ nanoparticles with porphyrins," J. Colloid Interf. Sci. Vol. 331, pp. 401-407, Mar. 2009.

[5] K. Wang, J. Yang, L. Feng, Y. Zhang, L. Liang, W. Xing, C. Liu C, "Photoelectrochemical biofuel cell using porphyrin-sensitized nanocrystalline titanium dioxide mesoporous film as photoanode," Biosens. Bioelectron. Vol. 32, pp. 177-182, Feb. 2012.

[6] C. Wang, G.M. Yang, J. Li, G. Mele, R. Słota, M.A. Broda, M.Y. Duan, G. Vasapollo, X. Zhang, F.X. Zhang, "Novel meso-substituted porphyrins: Synthesis, characterization and photocatalytic activity of their $\mathrm{TiO}_{2}$-based composites," Dyes Pigments. vol. 80, pp. 321-328, Mar. 2009. 
[7] T. Ma, K. Inoue, H. Noma, K. Yao, E. Abe "Effect of functional group on photochemical properties and photosensitization of $\mathrm{TiO}_{2}$ electrode sensitized by porphyrin derivatives," J. Photochem. Photobiol. A. Chem. Vol. 152, pp. 207-212, Sep. 2002.

[8] M.A. García-Sánchez, V. de la Luz, M.I. Coahuila-Hernández, F. Rojas-González, S.R. Tello-Solís, A. Campero, "Effects of the structure of entrapped substituted porphyrins on the textural characteristics of silica networks," J. Photochem. Photobiol. A. Chem. Vol. 223, pp. 172-181, Sep. 2011.

[9] M. Nakamura, T. Tatsumi, H. Tominaga, "Synthesis and Catalytic Capability of Zeolite-Encapsulated Iron and Manganese Tetramethylporphine Complexes," H. Bull. Chem. Soc. Jpn. Vol. 63, pp. 334-3336, Nov. 1990.

[10] E. Brulé, Y.R. de Miguel, "Supported metalloporphyrin catalysts for alkene epoxidation," Org Biomol Chem. vol. 4, pp. 599-609, Feb. 2006.

[11] A.D. Adler, F.R. Longo, F. Kampas, J. Kim, J. "On the preparation of. Metalloporphyrins," J. Inorg. Nucl. Chem. vol. 32, pp. 2443-2445, 1970.
[12] C.E. Díaz-Uribe, M.C. Daza, F. Martínez, E.A. Páez-Mozo, C. Guedes, E. Di Mauro, "Visible light superoxide radical anion generation by tetra(4-carboxyphenyl) porphyrin/ $\mathrm{TiO}_{2}$ : EPR characterization," J. Photochem. Photobiol. A. Chem. Vol. 215, pp. 172-178, Sep. 2010.

[13] M. Gouterman, G.H. Wagniere, L.C. Snyder, "Spectra of porphyrins. Part II: Four orbital model," J. Mol. Spectrosc. Vol. 11, pp. 108-127, 1963.

[14] W. Zheng, N. Shan, L. Yu, X. Wang, “UV-visible, fluorescence and EPR properties of porphyrins and metaIloporphyrins," Dyes Pigments. Vol. 77, pp. 153-157, Sep. 2008.

[15] S. Takagi, T. Shimada, T. Yui, H. Inoue, "High Density Adsorption of Porphyrins onto Clay Layer without Aggregation: Characterization of Smectite-Cationic Porphyrin Complex," Chem. Lett. Vol. 30, pp. 128-129, 2001.

[16] S.S. Cady, T. Pinnavaia, "Porphyrin intercalation in mica-type silicates," J. Inorg. Chem. Vol. 17, pp. 15011507, 1978. 